\title{
Chapitre I - Le contexte dans lequel évoluent les chemins de fer secondaires
}

Maurice Wolkowitsch

\section{CpenEdition}

\section{Journals}

Édition électronique

URL : https://journals.openedition.org/rhcf/1301

DOI : 10.4000/rhcf.1301

\section{Éditeur}

Rails \& histoire

\section{Édition imprimée}

Date de publication : 10 février 2004

Pagination : $24-40$

ISBN : 0996-9403

ISSN : 0996-9403

\section{Référence électronique}

Maurice Wolkowitsch, "Chapitre I - Le contexte dans lequel évoluent les chemins de fer secondaires », Revue d'histoire des chemins de fer [En ligne], 30 | 2004, mis en ligne le 30 août 2012, consulté le 22 avril 2022. URL : http://journals.openedition.org/rhcf/1301 ; DOI : https://doi.org/10.4000/rhcf.1301 


\section{Chapitre I \\ Le contexte dans lequel évoluent les chemins de fer secondaires}

ntre 1860 et 1940 la France connait de profondes mutations ;
la Première Guerre mondiale en accélère le rythme : la répartition des hommes, les structures de la société comme de l'économie se modifient. Les CFIL et les TVM sont conçus dans le cadre des départements. Entre l'élaboration et l'aboutissement de bien des projets, des années peuvent s'écouler; parallèlement l'évolution démographique se poursuit vers une double concentration géographique de la population au profit des villes et de quelques départements. $70 \%$ des Français vivaient en zone rurale en 1866, $56 \%$ en $1911,48 \%$ en $1936,25 \%$ en 1975, évolution retardée par rapport à d'autres pays industriels : dès 1900, l'Angleterre ne comptait que $25 \%$ de ruraux. La demande de transport en zone rurale, cible privilégiée des chemins de fer secondaires, était sensible à ces évolutions. Le contexte géographique et socioéconomique de la France en 1911 doit être précisé.

\section{Le contexte administratif et démographique}

\section{. Les départements}

La France est amputée de l'Alsace et d'une partie de la Lorraine ; les données concernant les anciens départements de la Moselle, du BasRhin et du Haut-Rhin ne figurent plus dans les documents français. Le territoire de Belfort érigé en nouvelle collectivité territoriale témoigne de l'existence passée du département du Haut-Rhin. Le département de la Corse n'est pas encore scindé en deux entités administratives distinctes. Enfin, la réforme de 1964 étant encore à venir, le département de la Seine est constitué de Paris et des arrondissements de Sceaux et Saint-Denis ; il inclut toutes les communes de la proche banlieue : il est enclavé dans la Seine-et-Oise, département qui est, à l'Est, limitrophe de la Seine-et-Marne. Ces derniers départements offrent toujours des vastes étendues spécifiquement rurales. Les conseils généraux n'étant pas touchés encore par la manie du changement, la fièvre touristique et les complexes d'infériorité, le département des Côtes-du-Nord n'est pas devenu Côtes-d'Armor, les départements de Loire, de Seine et Charente sont inférieurs avant d'être atlantique ou maritime, les Pyrénées et Alpes sont encore basses avant de devenir atlantiques ou de Haute-Provence. 


\section{. Une concentration géographique de la population}

Entre 1901 et 1911, 25 départements bénéficient d'une croissance de leur population, 8 s'enrichissent en 10 ans de $1 \%$ ou moins, 8 autres de 1,1 à 4,9 \%; 9 départements seulement présentent une augmentation significative de leur population : Nord : $5 \%$; Rhône : $8,5 \%$; Bouchesdu-Rhône : 9,6 \% ; Territoire de Belfort : 9,8 \% ; Pas-de-Calais : 11,8 \% ; Seine : $14 \%$; Seine-et-Oise : 15,5\% ; Meurthe-et-Moselle : 16,5\% ; Alpes-Maritimes : $21 \%$. La liste ne comporte aucun département à spécificité rurale et agricole dominante. De ces derniers la régression démographique est le lot, atteignant des taux supérieurs à $5 \%$ dans douze d'entre eux : Orne : $6 \%$; Tarn-et-Garonne : 6,6\% ; Gers : 7,3 \% ; Nièvre : 7,5 \% ; Lot : 9,2\%... Parallèlement, un mouvement d'urbanisation, maintes fois décrit, se poursuit sous deux formes. Les principales villes connaissent des taux de croissance élevés (Nice : $35 \%$; Nantes : $28 \%$; Grenoble : $16 \%$; Lyon : $14 \%$; Marseille : $12 \%$...), la région parisienne (Paris : 6,1\% ; Versailles $9 \%$, mais des villes beaucoup plus modestes se développent également (Albi : $13 \%$; Châteauroux : 6,9\%). Quelle que soit la forme d'urbanisation, le mouvement ne peut être favorable au développement du trafic des voyageurs sur les réseaux secondaires en zone rurale, sauf à envisager un nombre accru de voyages annuels accomplis par des ruraux. L'étude des trafics devrait permettre d'apporter des réponses à ces questions.

\section{. De grandes inégalités de superficies et de populations entre les départements}

Les départements offrent de grandes inégalités de superficies. Le département de la Seine avec $478 \mathrm{~km}^{2}$ est le moins étendu, suivi du Territoire de Belfort avec $624 \mathrm{~km}^{2}$, héritage de l'histoire ; hors ces cas particuliers restent 85 départements. Quatre ont moins de $4000 \mathrm{~km}^{2}$ : Rhône : 2856 ; Tarn-et-Garonne : 3718 ; Vaucluse : 3742 ; AlpesMaritimes : 3 761. Six départements, en partie montagneux, comptent entre 4000 et $5000 \mathrm{~km}^{2}$ : Ariège, Loire, Haute-Loire, Hautes-Pyrénées, Pyrénées-Orientales, Haute-Savoie. À l'opposé, seuls trois départements aquitains dépassent $9000 \mathrm{~km}^{2}$ : Landes : $9316 \mathrm{~km}^{2}$; Dordogne : 9330 ; Gironde : 9778 . Six autres disposent de superficies comprises entre 8000 et $9000 \mathrm{~km}^{2}$ (Marne, Isère, Corse, Saône-et-Loire, Aveyron, Côted'Or). 66 offrent des étendues comprises entre 5000 et $8000 \mathrm{~km}^{2}$.

La diversité est aussi sensible en matière de population. Le cas de la Seine avec 4154042 habitants dont 2881110 à Paris est particulier ; la croissance est rapide dans quelques villes de banlieue : Saint-Denis, Levallois, Boulogne... La Seine-et-Oise est sensible à l'influence parisienne, 
la Seine-et-Marne (363 000 habitants) en est encore préservée. Les autres départements offrent un très large éventail de population. Deux départements dépassent le million d'habitants (Pas-de-Calais : 1068 000, Nord : 1961000 ), six ont entre 700000 et 1 million d'habitants (Bouches-du-Rhône : 805000 ; Finistère : 809000 ; Seine-etOise : 817000 ; Gironde : 829000 ; Seine-Inférieure : 877000 ; Rhône 915 000). 12 départements, dont cinq dans l'Ouest, notamment en Bretagne, et d'autres connaissant un développement industriel (Isère, Loire, Puy-de-Dôme, Meurthe-et-Moselle...) ont entre 500000 et 700000 habitants. À l'opposé, six départements reçoivent un effectif inférieur à 200000 habitants (Ariège : 198000 ; Tarn-et-Garonne : 192000 ; Lozère : 122000 ; Basses-Alpes : 107000 ; Hautes-Alpes : 105000 ; Territoire de Belfort : 101 000). 60 départements se situent dans la fourchette comprise entre 200000 et 500000 habitants ; dans chaque cas, ces chiffres donnent une indication sur la clientèle potentielle des chemins de fer secondaires.

Le rappel de ces données semblait nécessaire parce qu'on ne peut éluder la question d'un rapport entre l'extension des réseaux secondaires, l'étendue du territoire et le nombre des hommes à desservir dans un département.

\section{Influence de l'évolution de la société sur le développement des réseaux secondaires}

Deux périodes doivent être distinguées, séparées par la Première Guerre mondiale. En 1860, les grandes compagnies de chemin de fer sont des puissances à l'égal des compagnies pétrolières aujourd'hui. Elles ont relié Paris à 16 ports de mer, à de multiples villes frontières hors celles des Pyrénées et du comté de Nice ; leurs rails s'arrêtent à Toulon, Limoges, Rennes, Bayonne et Perpignan. Elles sont une des forces en présence.

\section{. Première période 1860-1913}

\section{$>$ Les forces en présence}

\section{Les grandes compagnies}

Les grandes compagnies sont liées à la haute finance et aux industries de pointe de l'époque, la production houillère et la sidérurgie ; elles pratiquent un lobbying efficace, dirait-on aujourd'hui ; elles comptent toujours des parlementaires au sein de leur conseil d'administration et entretiennent des relations complexes avec les gouvernements. Tout au long de leur existence, elles sont menacées de rachat ; leur puissance est 
constamment dénoncée : en 1865, le baron Charles Dupin ${ }^{1}$ la comparait à celle des grands féodaux, terme qu'on retrouve en 1937 sous la plume de l'historien Marcel Blanchard ${ }^{2}$ pour décrire le partage territorial de la France défini par les conventions de Franqueville en 1859. Les compagnies bénéficiaient de concessions à monopole et du système de la garantie d'intérêt qui instituait une solidarité financière entre elles et l'Etat et assurait aux capitaux engagés dans les chemins de fer en France un rapport supérieur à celui enregistré à l'étranger : en 1877, $6 \%$ contre 4,5\% en Angleterre et 2,7 en Italie ${ }^{3}$. Malgré les pressions, les compagnies n'acceptaient à travers des conventions successives que des extensions très limitées de leur réseau. Les conventions Raynal de 1883 ont fait exception en y incluant des milliers de kilomètres de lignes en exploitation ou à construire, au titre du plan Freycinet, moyennant l'application d'une comptabilité particulière, pour ne pas imputer leur déficit probable sur les bénéfices tirés de l'exploitation des lignes rentables.

\section{L'opinion publique et l'exemple du Bas-Rhin}

L'opinion publique voit dans les compagnies l'expression de la volonté du capital parisien, voire étranger, indifférent aux intérêts économiques des provinces. Elle dénonce le monopole et réclame la concurrence avec la création de nouvelles compagnies ; ainsi les tarifs élevés, jugés injustifiés, baisseraient ; le contrôle et la réglementation des tarifs sont souhaités. Des pamphlétaires virulents réclament le tracé desservant leur clocher et s'indignent de la médiocrité des services offerts aux usagers, dépeints avec tant de verve par les caricatures de Daumier ou le théâtre de Labiche ${ }^{4}$. Un courant régionaliste composite s'affirme à travers les vœux des collectivités territoriales et des chambres de commerce, les prises de position de négociants et de petits notables attachés à la terre ; tous souhaitent un système de communications alliant voies d'eau et nouvelles compagnies de chemin de fer, conçu et dirigé par des personnalités issues de leurs milieux. Le monde rural dans sa diversité domine encore nettement pendant toute cette période. La société urbaine se diversifie avec l'essor du commerce, de l'industrie et de la banque. Les entreprises familiales dont certaines emploient plusieurs

1- Cité par F. Caron, Histoire des chemins de fer en France, tome I, 1740-1883, Paris, Fayard, 1997, $700 \mathrm{p}$.

2- M. Blanchard «La politique ferroviaire de Besançon 1844-1860», Bulletin de la Société languedocienne de géographie, 1937, p. 1-56.

3- A. de Foville, La Transformation des moyens de transport et ses conséquences économiques et sociales, Paris, 1880, $480 \mathrm{p}$.

4- Le théâtre de Labiche offre plusieurs scènes se déroulant dans des gares : Le Voyage de Monsieur Perrichon, 1860, et Les Chemins de fer. 
centaines de personnes se multiplient. Toutes ces activités requièrent apport de matières premières et accès aux marchés et étoffer le réseau ferré devient une exigence pressante à laquelle les élus ne sont pas insensibles. Les conseils généraux élaborent des projets ou réclament de nouvelles voies ferrées ou, comme celui du Bas-Rhin, passent à l'action.

Le cas de ce département mérite d'être analysé5. La Compagnie de l'Est y exploitait la ligne Strasbourg-Mulhouse tracée à travers la plaine rhénane peu peuplée, ignorant les petites villes et gros bourgs du piémont vosgien animé par l'agriculture et l'industrie textile; les relier par des embranchements à la grande ligne est devenu une demande des populations, refusée par l'Est. Les collectivités territoriales ont pris le projet en main en y associant les forces économiques locales ; soucieuses de s'inscrire dans la légalité, elles s'appuient sur le raisonnement suivant : «À notre époque où l'on parle beaucoup de décentralisation, on remarquera que la loi de 1836 a décentralisé de façon presque absolue les services de la voirie vicinale, puisque les ressources dont elle fait usage sont votées par les conseils généraux et municipaux et employées par les autorités départementales 6 . » Préfet, ingénieur des Ponts et conseil général décident d'un commun accord d'affecter, en 1858, une partie des crédits votés au titre de la loi de 1836 pour la construction de routes à celle de voies ferrées ; le conseil général assurera les terrassements. Ces responsables argumentent pour justifier cette décision prise de leur propre autorité : « La voie de fer n’est qu'une route perfectionnée quant à son tracé, ses pentes et ses courbes et cette route pourrait aussi bien recevoir un empierrement qu'un système de rails... ${ }^{7} \gg$. Une association est créée pour exploiter les lignes. Trois ans de négociations (18581861) précéderont la construction ; la mise en service est effective en 1864 entre Sélestat et Sainte-Marie-les-Mines, Haguenau et Niederbronn, Strasbourg et Molsheim et de la Barr ou Wasselone. L'exemple du BasRhin montre que les initiatives locales ne suscitent pas les mêmes difficultés que les projets des grandes compagnies : « les acquisitions de terrains se feront avec une facilité remarquable car presque toutes furent traitées à l'amiable et sans intervention du jury d'expropriation; au contraire les grandes compagnies étrangères aux localités ont à lutter

5- M.-H. Bierzy, «Les chemins de fer vicinaux », Revue des Deux-Mondes, 15 janvier 1866, p. 435-458.

6- Ibid., p. 437, l'auteur souligne que $83 \%$ des chemins de grande communication, 55 $\%$ des chemins d'intérêt commun, $30 \%$ des chemins vicinaux ordinaires sont déjà construits en 1863, ce qui justifie de pouvoir affecter une part des crédits à la construction de voies ferrées.

7- Ibid., p. 440. 
contre des prétentions souvent exorbitantes. On n'eut pas besoin non plus de réunir de grands ateliers d'ouvriers nomades qui jettent toujours plus ou moins le trouble dans un canton et y font hausser temporairement le prix habituel des salaires et des objets de consommation. Les terrassements furent exécutés par les prestataires comme s'il ne s'agissait que de chemins vicinaux ordinaires et aux époques de l'année où l'agriculture réclamait le moins de bras utiles ${ }^{8}$.» Ces avantages se sont traduits par un coût de construction au $\mathrm{km}$ particulièrement faible : $117000 \mathrm{~F}$ contre 432000 en moyenne pour les grandes compagnies; ces dépenses ont été couvertes pour 18,7\% par le département, 19,1\% par les communes dont les prestations consistaient essentiellement en cession de terrains communaux et en prestations en nature, par la compagnie concessionnaire pour 46,2\%?. L'État fut conduit à intervenir pour $16 \%$ pour permettre la réalisation du projet : il octroie à la société locale créée une subvention de $20000 \mathrm{~F}$ par km, pour que cette expérience de chemin de fer économique d'initiative locale soit conduite à son terme ; initialement le département et les communes concernées s'étaient engagés à réaliser les terrassements et les travaux d'art, tâches réservées à l'Etat par la loi de 1842 sur les chemins de fer; la démonstration était faite que la construction d'une voie à écartement normal pouvait être réalisée, pour une dépense de l'ordre de $120000 \mathrm{~F}$ le km, davantage en montagne, mais aussi que l'intervention de l'État s'était avérée indispensable. En définitive, la société financière locale remit les lignes en état de fonctionner à la Compagnie de l'Est, qui en assura l'exploitation, moyennant une garantie de recettes de $8500 \mathrm{~F}$ par an et par $\mathrm{km}$. Toutes les leçons de l'expérience $\mathrm{du}$ Bas-Rhin sont retenues par les législateurs.

\section{L'État}

L'État se trouve devant l'expression de ces volontés contradictoires que traduisent l'inertie des compagnies et le foisonnement désordonné des initiatives locales. Soucieux de l'équilibre financier des grandes compagnies et par ailleurs impuissant à les contraindre à allonger leur réseau, l'État octroie des concessions de chemins de fer à des nouvelles compagnies ou à des particuliers; le but est de construire des lignes destinées à assurer les transports de proximité que le roulage agonisant ne satisfait plus et à ouvrir des liaisons avec une gare de transit située sur un grand

8- Ibid., p. 443.

9- Les coûts de construction au $\mathrm{km}$ cités par Bierzy sont supérieurs à ceux donnés habituellement. 
réseau ; des concessions portant sur des parcours de 10 à $50 \mathrm{~km}$ ont été attribuées, sans porter ombrage aux grandes compagnies ; certaines, notamment le Nord, ont favorisé la réalisation de quelques projets.

Cette politique répondait partiellement aux revendications exprimées par les intérêts locaux. La création de relations transversales, dont seule la liaison Bordeaux-Marseille est achevée avant 1860, est exigée ; dès 1860, le conseil général de l'Eure demande d'unir Rouen à Orléans, le prolongement vers Châlons-sur-Marne est souhaité ultérieurement; un chemin de fer des Sables-d'Olonne à Montluçon par Tours et Châteauroux est proposé. Ces forces régionales voulaient surtout briser le monopole, créer la concurrence en inscrivant des lignes et réseaux dans les espaces enclavés entourés par les axes exploités par les grandes compagnies. Les personnalités locales poussaient à la création de compagnies et obtenaient des concessions qui, dans l'esprit de l'État, devaient s'en tenir à une vocation régionale : Compagnies des Charentes (1862), de Vendée (1863), des Dombes $(1864)^{10}$. Des concurrences au PLM se préparaient avec un itinéraire plus court de Lyon à Genève ou une nouvelle ligne de Lyon à Saint-Étienne.

Devant cette fièvre, l'idée se développe d'un nouveau type de chemin de fer qui pourrait être défini à l'exemple du réseau routier diversifié, de la route nationale à la route vicinale ; parallèlement, l'État juge indispensable d'encadrer ces velléités de constructions ferroviaires, tout en accordant certaines satisfactions aux représentants des intérêts locaux : la loi de 1865 portant création des chemins de fer d'intérêt local est une première réponse à ces initiatives.

\section{$>$ Le cadre juridique : de nouveaux types de chemins de fer}

Les dispositions fondamentales qui régissent les chemins de fer secondaires sont arrêtées entre 1865 et 1913 : la loi de 1865 est un texte de circonstance adopté sous la pression des événements ; ses imperfections sont corrigées par la loi de 1880, objet de véritables débats ; la loi de 1913 vise à l'unification de cette activité et à l'établissement de son encadrement plus strict sur le plan financier.

\section{La loi de $1865^{11}$}

Les CFIL peuvent être établis soit par les départements et les communes avec ou sans le concours des propriétaires intéressés, soit par le concessionnaire avec le concours des départements et des communes.

10- P. Protat, «Les Compagnies de chemins de fer secondaires 1859-1884», 2001, étude déposée à l'AHICF, p. 441.

11- Potiquet, Recueil des Lois et Ordonnances, décrets, règlements et circulaires, $1^{\text {te }}$ série $\mathrm{t}$. IX, $1^{\text {te }}$ partie, p. 283-284. Le texte des lois de 1865, 1880, 1913 est reproduit en annexe des actes du colloque de Châteauroux, RHCF 24-25, p. 410-433. 
Le conseil général arrête le tracé, le mode et les conditions de construction, les traités et les dispositions nécessaires à l'exploitation... Les ressources créées pour le développement des routes vicinales peuvent être affectées à la construction d'un CFIL. La loi de 1845 sur la police des chemins de fer s'applique au CFIL, mais le préfet peut dispenser de l'obligation d'établir des clôtures et des barrières aux passages à niveau. Des subventions sur les fonds du Trésor peuvent être accordées pour la construction des CFIL, elles sont fonction de l'effort financier et des ressources fiscales du département concerné.

Mais ces libertés accordées aux pouvoirs locaux ont leurs limites; l'État a le dernier mot, il est présent aux diverses étapes auxquelles sont soumis les projets : "L'utilité publique est déclarée et l'exécution est autorisée par décret délibéré en Conseil d'État sur le rapport des ministres de l'Intérieur et des Travaux publics. Le préfet approuve les projets définitifs, après avoir pris l'avis de l'ingénieur en chef, homologue les tarifs et contrôle l'exploitation » (Article 2).

Une situation confuse se développe entre 1865 et le début des années quatre-vingt. L'État accorde indifféremment des concessions d'intérêt local ou d'intérêt général à de mêmes compagnies : entre 1865 et 1882, les compagnies secondaires ont reçu en concession $56 \%$ de lignes d'intérêt local, $44 \%$ de lignes d'intérêt général ; celle des Charentes associe respectivement 44 et $730 \mathrm{~km}$, celle des Dombes 259 et 166 ; de grandes compagnies aussi bénéficient de concessions de lignes d'intérêt local (Nord : $315 \mathrm{~km}$; Orléans : 96). Les conseils généraux, favorables à la concurrence et chargés de définir le mode de construction, s'en tiennent aux normes retenues pour les lignes d'intérêt général, et négligent les possibilités offertes par la voie étroite ${ }^{12}$.

Le choix de l'écartement normal permettait en principe l'interopérabilité entre les lignes d'intérêt général et local : les grandes compagnies reprochaient aux autres le mauvais entretien de leurs matériels et de leurs voies et freinaient le plus possible une utilisation commune. Ce choix favorisait les concessionnaires plus soucieux d'opérations financières que de la construction des lignes, ceux aussi dont l'objectif à terme était le rachat par les grandes compagnies, l'intégration étant simplifiée par la communauté d'écartement; ces politiques s'appuyaient sur la simple menace de créer des concurrences, tel le projet avorté du Calais-Marseille soutenu par Delahante ou sur d'authentiques réalisations de réseaux en jouant sur les possibilités offertes par les lignes interdépartementales permettant de proche en proche

12- P. Protat, ouvr. cité 
des liaisons lointaines : le cas des deux réseaux de l'illustre Philippart (Nord-Est et Ouest, de Poitiers à Saumur et Bressuire) $)^{13}$ est symbolique d’une époque. D’autres dirigeants, Jeancard, Mangini étaient avisés ; le second a su revendre au bon moment les concessions de la Compagnie des Dombes au $\mathrm{PLM}^{14}$. Ainsi s'effondraient les espoirs de briser les monopoles des grandes compagnies qui avaient été à l'origine de la création des compagnies secondaires.

La loi de 1865 n'a pas atteint pleinement ses objectifs : elle n'a ni mis de l'ordre dans la construction des chemins de fer, ni réussi à imposer un deuxième type de chemin de fer dont l'établissement et l'exploitation auraient été moins onéreux. Parallèlement à l'élaboration du plan Freycinet, l'adoption d'une nouvelle loi sur les CFIL est prévue dans le double but d'en favoriser le développement, tout en en contenant les dérives financières.

\section{La loi de $1880^{15}$}

La loi de 1880 reprend certaines dispositions prévues en 1865, telle la dispense préfectorale d'établir des clôtures le long de la voie et des barrières aux passages à niveau. Surtout, elle renforce la tutelle de l'État, définit les pouvoirs du concédant, fixe des conditions de financement propres à faire obstacle à la spéculation, traite dans un chapitre des CFIL, dans un autre d'un troisième type de chemin de fer de construction et d'exploitation plus économiques que le CFIL, jouissant d'une liberté administrative toute relative : le tramway.

L'intervention des autorités politiques et administratives à chaque stade de la vie d'un CFIL souligne son degré de dépendance vis-à-vis de l'État. Les procédures prévues pour l'établissement d'une ligne par la loi de 1865 sont maintenues, mais en plus l'avis du Conseil général des Ponts et Chaussées est obligatoire. La loi de 1880 institue la possibilité pour le ministre des Travaux publics, sur proposition du préfet et après avis du Conseil des Ponts, d'imposer à un conseil général une seconde délibération, dans les deux mois suivant la première. Le Conseil d'État délibère sur les décrets prévoyant une augmentation des tarifs, une cession ou une fusion de concession, un changement de concessionnaire, la substitution de l'exploitation directe à l'exploitation par concession. Le ministre statue au sujet des lignes interdépartementales si un désaccord existe entre conseils généraux ; il homologue le montant des taxes perçues

13- F. Caron, ouvr. cité, p. 451-460.

14- P. Protat, ouvr. cité, p. 24-25.

15- Journal officiel de la République française, 11-12 juin 1880, p. 227-233. 
(péages et tarifs) lorsqu'elles sont communes à plusieurs lignes ; il prononce la déchéance en cas d'inexécution d'une concession ; il peut décider de classer dans le domaine public toute voie ferrée à toute époque, moyennant l'indemnisation du concessionnaire. Un cahier des chargestype approuvé par le Conseil d'État s'impose au concessionnaire ; celui-ci peut demander des dérogations, soumises au Conseil d'État et inscrites dans la loi d'approbation du projet. Le concessionnaire doit adresser au ministre des Travaux publics et au préfet tous les trois mois le compte rendu détaillé des résultats d'exploitation (dépenses d'établissement et d'exploitation, résultats bruts).

Les concédants voient préciser leurs pouvoirs. Ils peuvent, sauf inscription dans l'acte de concession, octroyer des concessions concurrentes sans qu'un concessionnaire en titre puisse s'y opposer, autoriser l'embranchement d'une nouvelle voie sur une voie existante, permettant à une nouvelle entreprise d'utiliser moyennant péage une ligne concédée en service, décider du rachat d'une concession, également de la modification ou de la suppression d'un tracé.

Le volet financier, essentiel, fixe les conditions de financement et d'attribution des aides de l'État. Désormais le versement de $80 \%$ du capital-actions, son utilisation pour l'acquisition des terrains et divers approvisionnements ou son dépôt en cautionnement est obligatoire avant toute autorisation d'émission d'obligations accordée par le ministre des Travaux publics après avis du ministre des Finances. Le montant des obligations ne pourra dépasser celui du capital-actions, celui-ci fixé à la moitié au moins des dépenses nécessaires à l'établissement et à l'ouverture de la ligne. Après versement du capital-actions, dont $50 \%$ utilisés comme prévu, le concessionnaire peut procéder à une émission anticipée d'obligations sous condition de déposer les fonds à la Caisse des dépôts et consignations ; une autorisation formelle du ministre des Travaux publics permet seule au concessionnaire de les utiliser. On mesure les précautions prises pour en finir avec les concessionnaires peu soucieux d'exécuter les travaux.

Une même rigueur est observée dans l'attribution des aides de l'État pour éliminer les spéculateurs et la chasse aux concessions : on substitue aux subventions en capital une subvention versée par annuités. La subvention de l'État est accordée, en cas d'insuffisance de produit brut, pour couvrir les dépenses d'exploitation plus $5 \%$ par an du capital de premier établissement, et sous la condition d'une contribution égale des collectivités territoriales intéressées. La subvention est de $500 \mathrm{~F}$ par km exploité, plus le quart de la somme nécessaire pour élever la recette brute annuelle à $10000 \mathrm{~F}$ par $\mathrm{km}$ pour les lignes à écartement 
normal, $8000 \mathrm{~F}$ par $\mathrm{km}$ pour les lignes à voie étroite. L'État suspend son aide lorsque la recette brute atteint une limite définie. La loi de Finances fixe chaque année le montant des subventions que l'État peut accorder aux CFIL, dans la limite de 400000 F par département. L'État peut exiger sur une ligne subventionnée des services gratuits ou des réductions de prix.

La loi institue un nouveau type de chemin de fer: le tramway hippomobile ou à moteur mécanique ; son originalité est d'utiliser les routes et les rues comme assiette de la voie, ne les quittant que pour de brèves déviations imposées par le site. La rareté des ouvrages d'art favorise les économies de construction. Le tramway apparait comme un CFIL simplifié ; il peut être réservé aux voyageurs ou aux voyageurs, bagages et messageries : cette catégorie circule essentiellement dans les milieux urbains et périurbains. Les tramways pour voyageurs et marchandises (TVM) desservent au départ d'une ville ou d'une gare de transit avec une ligne d'intérêt général, comme les CFIL, des zones rurales et bénéficient comme eux de subventions d'État, mais à un moindre niveau, $6000 \mathrm{~F}$ par $\mathrm{km}$.

Beaucoup d'articles de la loi visent les TVM et les CFIL, particulièrement ceux fixant les conditions d'attribution des aides de l'État et de financement des projets, l'obligation de consulter les conseils généraux ou municipaux n'ayant pas à statuer sur la concession d'une ligne traversant leur territoire. Les modalités d'application peuvent être particulières : pour synthétiser, notons que, pour les TVM, le préfet se substitue souvent au ministre; le décret suffit là où la loi intervient pour les CFIL ; on le constate pour l'homologation des tarifs ou l'approbation des dérogations au cahier des charges-type. Cependant les procédures fondamentales conduisant à l'exécution d'un projet sont identiques, garantissant le même contrôle de l'État.

La loi comporte aussi des dispositions propres aux TVM souvent liées à l'utilisation du réseau routier. La concession est accordée par l'État pour l'emprunt des routes nationales, le département pour l'emprunt des routes départementales, de grande communication ou d'intérêt commun, par la commune pour toute ligne ne débordant pas son territoire et tracée uniquement sur des chemins vicinaux ordinaires et des chemins ruraux. Le département peut accorder une concession à l'État ou à une commune, une commune à l'État ou à un département, dans tous les cas avec une faculté de rétrocession. L'expropriation des terrains nécessaires à l'élargissement des chemins vicinaux et des déviations est prévue. Les concessionnaires sont dispensés de l'impôt sur les voitures et bêtes de trait utilisées exclusivement pour l'usage du 
tramway; ils ne peuvent être soumis au droit de stationnement exigible par les collectivités territoriales, sauf si une clause le prévoit dans l'acte de concession. À l'expiration de la concession, le concessionnaire peut être contraint à supprimer la voie ferrée et à remettre en l'état la viabilité de la voie publique ; cette disposition souligne le caractère éventuellement éphémère du TVM face au CFIL, prévu pour durer et dont le concessionnaire doit remettre au concédant les voies en bon état d'entretien. Enfin les concessionnaires de TVM doivent respecter les conditions spécifiques de construction et d'exploitation imposées par l'utilisation du domaine public, notamment les règlements propres à permettre la coexistence entre les divers usagers de la route.

\section{La loi du 31 juillet $1913^{16}$}

La loi unifie les dispositions applicables à l'établissement et à l'exploitation par les départements des CFIL et TVM et par les communes des TVB et des TV désignés sous le nom de voies ferrées d'intérêt local (VFIL). La loi précise les dispositions antérieures et en définit de nouvelles : elle s'organise en quatre titres.

La déclaration d'utilité publique et l'approbation des projets sont précédées d'un avant-projet précisant les localités à desservir, la tarification maximale prévue, le trafic probable, les voies et moyens pour y répondre, le montant de la subvention sollicitée de l'État. Ce texte accompagné des rapports de l'ingénieur en chef du département et du chef du service du contrôle départemental des chemins de fer d'intérêt local est transmis par le préfet au ministre des Travaux publics, qui consulte ses collègues de l'Intérieur et des Finances ; la mise à l'enquête est acquise par leur accord ; un décret délibéré en conseil des ministres après avis du Conseil d'État est nécessaire en cas de désaccord.

L'enquête faite, le conseil général arrête les dispositions et traités nécessaires à la construction et l'exploitation. Après la déclaration d'utilité publique, le préfet soumet le projet au conseil général qui statue définitivement; il ne peut imposer une second délibération. L'exécution est autorisée par une loi en cas de subvention d'État, par un décret dans les autres cas.

Si un département souhaite prolonger une ligne dans le département voisin pour permettre une meilleure exploitation, il peut prendre tous les frais à sa charge; si le voisin refuse le passage, l'enquête peut être autorisée par un décret délibéré en Conseil d’État.

16- Journal officiel du 31 juillet 1913, p. 268-280. 
Le régime financier vise au renforcement du contrôle du financement des VFIL déjà amorcé en 1880. Huit articles traitent des subventions de l'État. Pour chaque concession, la loi déclarative d'utilité publique précise : «le maximum des sacrifices de l'État ${ }^{17}$, et énonce les conditions d'octroi (capital ou annuités) et d'utilisation des subventions. La subvention ne peut plus couvrir une insuffisance d'exploitation ; elle ne peut être versée avant justification d'une dépense au moins double en achat de terrains, travaux, approvisionnements sur place, ou dépôts de cautionnement, clauses prévues en 1880 seulement pour les émissions d'obligations. L'article 19 rappelle les droits de l'État à une participation à l'excédent de recettes sur les dépenses d'exploitation d'une ligne bénéficiaire d'une subvention, sauf si celle-ci est inférieure à $25 \%$ du capital d'établissement, cas où l'État peut renoncer à ses droits. Contrairement aux règles uniformes établies en 1880, le partage entre État, département, commune proportionnellement à leur subvention et le concessionnaire est établi dans chaque cas et figure dans l'acte de concession.

Neuf articles arrêtent de nouvelles mesures, visant toutes à sélectionner des concessionnaires offrant le maximum de garanties. La loi fixe les méthodes de calcul des dépenses d'exploitation ${ }^{18}$, institue un fonds de réserves pour grosses réparations, renouvellement de la voie et du matériel. Avant la déclaration d'utilité publique une caution élevée doit être versée ${ }^{19}$; avant la signature de la concession, une somme égale à $20 \%$ du capital de premier établissement doit être engagée ${ }^{20}$; la part versée par le concessionnaire en cours de travaux doit à tout instant être égale à $20 \%$ des dépenses faites pour l'ensemble du réseau. Enfin la loi impose pour toute exploitation de VFIL la constitution d'une société anonyme ; en cas de concession à une personne physique, celle-ci doit dans les six mois constituer une société dont elle reste solidaire dix ans ; le conseil d'administration doit être composé d'une majorité de Français. Le temps des pionniers est fini.

17- La subvention est calculée d'après les charges réelles du département correspondant aux dépenses de premier établissement augmentées des dépenses pour travaux complémentaires pendant les 10 premières années d'exploitation. La part de la subvention varie entre 10 et $75 \%$ en fonction de normes précises (cf. art. 14).

18- Le calcul pouvait reposer sur le montant réel ou sur une formule prenant en compte les recettes d'exploitation, le nombre des trains, l'importance et la nature des trafics.

19- $1 / 20^{\mathrm{e}}$ des dépenses de $1^{\text {er }}$ établissement pour les cinq premiers millions, $1 / 40^{\mathrm{e}}$ audelà.

20- Peuvent être pris en compte les capitaux déjà dépensés pour d'autres voies ferrées qui constitueraient avec la nouvelle ligne un réseau groupé dans une même exploitation départementale. 
Les obligations imposées aux concessionnaires doivent figurer dans les traités les liant aux concédants. Le cahier des charges doit préciser les conditions dans lesquelles le concessionnaire participe à l'entretien des voies publiques empruntées et au transport du trafic postal. Les frais de contrôle des activités des VFIL, déjà à la charge des concessionnaires, sont définis dans le cahier des charges et soumis à un prélèvement maximum de $5 \%$ au bénéfice du Trésor. Pour s'assurer de la remise des voies ferrées au concédant en bon état, les départements peuvent dans les cinq années qui précèdent la fin de la concession exiger des travaux et des achats de matériel; ils peuvent placer sous séquestre le fonds de réserve et prendre l'initiative des travaux et achat en cas de carence du concessionnaire ; ces dispositions sont plus contraignantes que celles de la loi de 1880.

Diverses dispositions sont prévues. Le droit de timbre perçu pour les expéditions de marchandises circulant uniquement sur les voies de VFIL établies par les départements est uniformément fixé à 25 centimes, sauf pour les TVM en service où le droit réduit de 10 centimes est maintenu. Un article entier traite de la desserte des quais des ports maritimes et de navigation intérieure. Des règlements d'administration publique doivent déterminer les conditions d'application des mesures évoquées (calcul des recettes et dépenses, niveau et versement des subventions...), mais aussi les dispositions devant présider à l'exploitation directe par des départements ou communes. Une nouveauté à souligner est l'article 48 qui exige que le cahier des charges ou la convention annexés à l'acte déclaratif précise les conditions de travail et de retraite des personnels. Le dernier article abroge la loi de 1880, tout en maintenant pour les voies en service les droits de propriété et les rapports financiers entre État, concédants, concessionnaires et rétrocessionnaires. Les départements qui déclareront accepter que toutes leurs voies ferrées subventionnées ${ }^{21}$ ou non soient soumises à la nouvelle loi seront substitués à tous les droits de l'État sur les lignes ou réseaux qu'il leur a concédés. L'État conserve ses droits à la participation dans l'excédent des recettes.

Peu d'articles de la loi s'appliquent aux seules lignes d'initiative communale, généralement de TV. Le conseil municipal exerce les attributions confiées au conseil général après que celui-ci renonce à réaliser le projet ; l'approbation préfectorale n'est pas nécessaire. Toutes les procédures prévues pour les lignes départementales s’appliquent aux

21- Les départements ayant déjà obtenu des subventions ne pourront en obtenir de nouvelles qu'après avoir souscrit à la déclaration. L'application de la loi interviendra lors de l'expiration ou du remaniement des contrats de concessions en cours. 
lignes communales, de l'avant-projet à la mise à l'enquête sur le tracé proposé, l'emplacement des stations, les conditions de construction et d'exploitation ; le respect d'un cahier des charges-type est imposé. Une ligne débordant les limites communales pour en améliorer l'exploitation peut, soit être construite aux frais de la commune qui a établi le projet moyennant certaines autorisations, soit être l'œuvre d'un syndicat de communes, constitué dans ce but au titre de la loi du 22 mars 1890 ; dans ce cas, le comité et le président du syndicat assument les pouvoirs du conseil municipal et du maire. Cette possibilité montre l'adaptation de la loi à la formation d'agglomérations urbaines. Dans tous les cas l'avis de la commission départementale doit figurer au dossier.

L'intérêt de cette loi réside certes dans son contenu, mais tient aussi à l'année de son adoption; en 1913, on entrevoyait encore des perspectives d'extension du réseau; nul ne songeait que l'automobile et le camion allaient dans la décennie suivante amorcer une concurrence qui menacerait l'existence même des réseaux de VFIL.

\section{. Deuxième période 1914-1963}

Pendant la Première Guerre mondiale, le réseau des VFIL n’a pas évolué ; les lignes proches du front comme celles du Meusien entre Verdun, Bar-le-Duc et Revigny ont été largement utilisées ; pour lui permettre de jouer son rôle, des matériels ont été réquisitionnés à l'arrière jusqu'en Lozère, en Charentes, auprès d'une vingtaine de compagnies $^{22}$. En 1918, les compagnies de chemins de fer secondaires étaient dans une situation très difficile. Les projets d'allongement des réseaux arrêtés par le conflit militaire n'ont que partiellement été repris. Les articles de la loi de 1913 fixant de nouvelles exigences en matière financière et instituant l'obligation de faire figurer des clauses sociales dans le cahier des charges ont conduit des entrepreneurs à renoncer.

La guerre a entrainé des conséquences sur la totalité de la société.

Le monde agricole est touché dans ses forces vives ; les listes interminables figurant sur les monuments aux morts de chaque village en sont la traduction. L'absence de ces hommes jeunes retarde l'évolution de l'agriculture dans de nombreuses régions. L'autoconsommation est courante; les achats de biens de consommation sont limités. La commercialisation des produits de la terre, la mécanisation et la motorisation, l'utilisation d'engrais progressent lentement. Cet immobilisme se transmet au milieu rural dans sa globalité, notamment au commerce local. Cette économie ne peut conduire à une progression du trafic pour les VFIL.

22- P. Puig, «Le Meusien : utilisation d'une voie métrique par l'Armée française, 19141918 », in " Armées et chemins de fer en France », Revue d'bistoire des chemins de fer, $\mathbf{n}^{\circ} 15$ (automne 1998), p. 88-116. 
Les industries dispersées en ateliers réduits, parfois même à domicile, les entreprises familiales déclinent avant de disparaitre ; les banques locales survivent rarement à la crise de 1929 ; leur sort se règle entre faillite et absorption par les grands réseaux nationaux. Ces activités avaient fait naître, à côté des propriétaires fonciers, ce milieu dynamique qui avait soutenu et souvent réalisé les chemins de fer secondaires; ces notables provinciaux ont perdu leur influence, ils ont disparu ou, le plus souvent, comptent sur le camion et sur l'automobile pour satisfaire leur demande de transport. Les administrateurs des compagnies secondaires se tournent, tel Verney, vers le transport routier ; simple réplique des années 1840-1850 où un administrateur des Messageries royales entrait au conseil d'administration de la Compagnie des chemins de fer du Centre et trois dirigeants d'entreprises de roulage à la Compagnie des chemins de fer de Tours à Nantes. L'atmosphère dans laquelle sont nés et celle dans laquelle sont morts les chemins de fer secondaires se traduit bien dans ces deux exigences proclamées par des ingénieurs à 70 ans de distance, même si cela vise les grandes lignes : «il faut transformer tous les chemins de grande communication en voie ferrée » et «il faut déferrer toutes les lignes à double voie pour les transformer en routes modernes ». Les lobbies efficaces ne sont plus ceux qui soutiennent les chemins de fer, mais : «l'automobile n'a pas seulement le prestige qui s'attache aux choses nouvelles et la faveur du public ; elle est soutenue par toute la puissance du capital et par la protection des plus considérables, des mieux armées, des plus écoutées des industries modernes : la construction automobile, la métallurgie, les carburants ${ }^{23}$. »

La législation sur la coordination des transports théoriquement destinée à discipliner la concurrence entre le rail et la route par des échanges de trafic est un échec ${ }^{24}$; elle ne prend pas en compte par ailleurs les chemins de fer secondaires qui ne sont pas sous la tutelle de l'État. Chaque conseil général dans son département doit exercer sa responsabilité. La concurrence est très vive : les autocars sont plus rapides, les camions évitent les ruptures de charge au contact des voies métriques et normales. Les conseillers généraux sont placés devant des déficits croissants, soumis à des pressions politiques et économiques. Ils siègent dans les comités techniques départementaux où ils exigent le maintien des lignes déficitaires des grandes compagnies : les mêmes

23- H. Cavaillès, La Route française, son histoire, sa fonction, Paris, 1946, 365 p.

24- M. Wolkowitsch, «Les facteurs de la concurrence entre les modes de transports aux XIX ${ }^{e}$ et XX ${ }^{e}$ siècles », in "Chemins de fer en temps de concurrences. Choix du XIX ${ }^{e}$ siècle et débats actuels ", Actes du $6^{e}$ coll. de l'AHICF, Revne d'histoire des chemins de fer, $\mathrm{n}^{\circ}$ 16-17 (1997), p. 47-64. 
dans les assemblées départementales votent sans états d'âme la mort des compagnies secondaires, dont la survie aurait exigé un appel aux finances dont ils avaient la gestion. Les votes ont été acquis au cours des années trente à l'unanimité ou à une majorité écrasante. Les opposants étaient si rares qu'à la mort d'un confrère, un conseiller général rappelait son combat sur sa tombe : «Je le revois encore à une des dernières sessions, malade déjà mais se multipliant pour sauver sa ligne et prenant par la veste chacun de ses collègues pour forcer une conviction qui se dérobait ${ }^{25}$.»

Sans remonter au Guide des chemins de France publié par Charles Estienne en 1552, décrivant $25000 \mathrm{~km}$ d'itinéraires, certains à l'état de sentiers ou de pistes, les routes nationales héritées de l'œuvre des intendants du XviII siècle ont en 1930 autour de 170 ans. Une statistique établie au 31 décembre 1863 souligne que, grâce à l'application de la loi du 31 mai 1836, la France est dotée de 110000 km de chemins vicinaux ordinaires, 43000 de chemins d'intérêt commun, de 69000 de chemins de grande communication. Les chemins de fer secondaires ne peuvent se réclamer d'un si lointain passé, sauf ceux encore en activité : le chemin de fer du Blanc-Argent est en 2003 en service depuis 104 ans entre Romorantin et Salbris, 103 ans entre Romorantin et Luçay-leMâle ; Nice est reliée à Digne depuis 94 ans ; le Train jaune de Cerdagne de Villefranche-Vernet-les-Bains a gagné Bourg-Madame en 1910, Montlouis en 1911, mais Latour-de-Carol seulement en 1927, il y a 76 ans. Les lignes abandonnées, généralement déclassées, n’offraient pas en 1930 une même longévité, à l'exception de la ligne MontpellierPalavas, célébrée par Dubout, qui a résisté de 1872 à 1968 ; le réseau des Chemins de fer départementaux en Indre-et-Loire a été exploité suivant les lignes entre 42 et 64 ans, celui des Tramways de l'Indre entre 32 et 35 ans ; la ligne interdépartementale entre Redon et Guer a fonctionné 13 ans (1924-1937). Il serait vain de multiplier les exemples; à titre de synthèse, notons que les durées les plus fréquentes se situent entre 30 et 70 ans. Le domaine des chemins de fer secondaires est celui de l'éphémère. Cela n'était pas sans conséquence pour les entreprises qui en assuraient l'exploitation.

25- J. Finsterwald et C. Bouchaud, « Les Chemins de fer vicinaux de la Haute-Saône de l'extension (1878-1912) au déclin (1918-1938) ", Chemins de fer régionaux et urbains, $\mathrm{n}^{\circ} 287\left(2001, \mathrm{n}^{\circ} 5\right)$, p. 3-15. 